\title{
A taxonomic study on the genus Ettchellsia Cameron, with descriptions of three new species (Hymenoptera, Megalyridae, Dinapsini)
}

\author{
Toshiharu Mita ${ }^{1, \dagger}$, Scott R. Shaw ${ }^{2, \ddagger}$ \\ I Laboratory of Entomology, Faculty of Agriculture, Tokyo University of Agriculture, 1737 Funako, Atsugi-shi, \\ Kanagawa 243-0034 JAPAN 2 University of Wyoming Insect Museum, Department of Ecosystem Science and \\ Management (3354), University of Wyoming, 1000 East University Avenue, Laramie, Wyoming 82071, U.S.A. \\ † urn:lsid:zoobank.org:author:6CC49F2C-C91C-463C-9C2D-8269588E1A6A \\ †urn:lsid:zoobank.org:author:B4DCD509-07A6-45E1-AF25-296430A49EED \\ Corresponding author: Toshiharu Mita (tkp_ex@hotmail.com) \\ Academiceditor:C.vanAchterberg|Received25October2012|Accepted11 December2012|Published21 December2012 \\ urn:lsid:zoobank.org:pub:D4519DDA-6E5F-43DA-8876-FE0434A305E5 \\ Citation: Mita T, Shaw SR (2012) A taxonomic study on the genus Ettchellsia Cameron, with descriptions of three new \\ species (Hymenoptera, Megalyridae, Dinapsini). ZooKeys 254: 99-108. doi: 10.3897/zookeys.254.4182
}

\section{Abstract}

Three new species of Ettchellsia Cameron, namely, E. ignita sp. n. from Peninsular Malaysia and Borneo, E. nigripes sp. n. from Sulawesi and E. reidi sp. n. from Borneo are described and illustrated. A key to the species of Ettchellsia is provided based on females.

\section{Keywords}

Taxonomy, parasitic wasps, South East Asia 


\section{Introduction}

Cameron (1909) described a remarkable new wasp genus from Borneo that he dedicated to his housekeeper, Mary Ettchells. Cameron found the insect so unusual that he struggled to place it to family, and debated if it should be placed into a new family. Subsequent authors (Baltazar 1961; Shaw 1988, 1990; Vilhelmsen et al. 2010) have clarified the placement of Ettchellsia Cameron in the family Megalyridae. Ettchellsia Cameron 1909 is a small genus comprising three described species (Cameron 1909; Baltazar 1961; He 1991). Ettchellsia species show an Indomalayan distribution, i.e., E. piliceps Cameron occurring in Borneo, E. philippinensis Baltazar occurring in Philippines and E. sinica occurring in Yunnan, China. Shaw (1990) defined Ettchellsia as a monophyletic genus based on three synapomorphies: the rugose hind tibia, unique pattern of propodeal carinae (as in Figs 10-12), and smooth posterior border of the mesopleuron, without a row of foveae separating the mesopleuron and metapleuron. Shaw (1990) also examined specimens from Thailand and Viet Nam, which he regarded as range extensions and variations of Ettchellsia piliceps Cameron. One specimen from Taiwan was not placed to species because it was a male. Vilhelmsen et al. (2010) also referred to unidentified species of Ettchellsia from Taiwan and Thailand. According to recent phylogenetic studies, the genus Dinapsis Waterson (distributed in the Afrotropical region) was strongly suggested to be the closest relative of Ettchellsia (Shaw 1990; Vilhelmsen et al. 2010). Ettchellsia species are quite rare; however, recently some specimens were obtained from Peninsular Malaysia, Sulawesi and Kalimantan. They were identified by us as three different new species. These findings and recent taxonomic works on the genus Carminator Shaw, the other genus of the family found in Asia (Mita et al 2007; Mita and Konishi 2011) may imply the megalyrid species diversity of the Indomayan region is still not clarified well. The biology of Ettchellsia has remained unknown, and no distributional areas are reported except for type localities. It is almost the same case with Carminator. Based on a knowledge of other Megalyridae, Ettchellsia is presumed to be an idiobiont ectoparasitoid, probably attacking beetle larvae (Shaw 1990). Southeast Asia is a key area to understanding the complicated evolutionary history of the family Megalyridae because the sister-group pairs of both Ettchellsia (Dinapsis Waterson: Afrotropical) and Carminator (Cryptalyra Shaw: Neotropical) occur in far separated areas (Shaw 1990; Vilhelmsen et al. 2010). Further findings from Southeast Asia will help the understanding of the evolutionary history of the Megalyridae.

\section{Materials and methods}

The morphological terms used in the descriptions follow Shaw and van Noort (2009) and Vilhelmsen et al. (2010). Photo images were produced using Leica Application Suite (Leica Microsystems) and Combine ZM software (Alan Hadley, www.hadleyweb. pwp.blueyonder.co.uk). Line drawings were made using a drawing tube attached to a 
binocular microscope. The depositories of the types are as referenced after the collection data.

\section{Taxonomy}

\section{Genus Ettchellsia Cameron, 1909}

http://species-id.net/wiki/Ettchellsia

Ettchellsia: Cameron 1909: 208; Baltazar 1961: 219; He 1991: 475

Type species. Ettchellsia piliceps Cameron, 1909 (monotypic).

Diagnosis. Eye margined posteriorly by post-orbital orbital carina; posterior border of mesopleuron smooth, without row of foveae; propodeum bearing unique pattern of longitudinal carinae (Figs 12-14); fuscous banding pattern present on fore wing (Figs 1, 4, 7) or wing entirely fuscous; fore wing with RS between RS+M and r-rs tubular (Fig. 4); apical part of RS tubular, arched toward stigma (Fig. 4); $\mathrm{M}+\mathrm{Cu}$ and $\mathrm{Cu} 1$ spectral (Fig. 4); hind coxa bearing longitudinal carina; hind tibia rugose (Figs 1, 4, 7).

Detail generic character states were discussed by Shaw (1990) and Vilhelmsen et al. (2010).

\section{Key to the females of the species of Ettchellsia}

1 Ocellar triangle smooth, without a longitudinal row of punctures; smooth crescent-shaped depression present on outer margin of each ocellus; wings mostly clear except with some infumation medially and apically, but lacking distinct dark bands.

E. philippinensis Baltazar

- Ocellar triangle sculptured with at least one median longitudinal row of punctures (Fig. 2); crescent-shaped depressions absent near ocelli, or depressions irregularly foveate, not smooth; wings with three or four distinct dark bands 2

2 Vertex strongly flattened, appearing flat in lateral view (Fig. 11); mesonotum more weakly setose, setae not covering surface

E. piliceps Cameron

- $\quad$ Vertex well-developed and convex, appearing round in lateral view (Fig. 10); mesonotum medially and posteriorly with thick setae largely covering surface.

- $\quad$ Mesoscutum not humped (Fig. 6)

4 Median region of propodeum anteriorly strongly narrowed (as in Fig. 12); hind tibia and basitarsi dorsally with long black erect setae, many of which are 
longer than the width of the hind tibia; metasoma reddish brown (Fig. 1)....

E. ignita Mita \& Shaw, sp. n.

- $\quad$ Median region of propodeum not anteriorly strongly narrowed; hind tibia and basitarsi dorsally with long erect white (not black) setae, most of which are shorter than the width of the hind tibia; metasoma black (as in Fig. 4) ...

E. sinica $\mathrm{He}$

Median region of propodeum medially narrowed (Fig. 14); vertex between posterior ocellus and eye rugose (Fig. 8) E. reidi Mita \& Shaw, sp. n.

- $\quad$ Median region of propodeum anteriorly weakly narrowed (Fig. 13); vertex between posterior ocellus and eye mostly smooth (Fig. 5)

E. nigripes Mita \& Shaw, sp.n.

\section{Ettchellsia ignita Mita \& Shaw, n. sp.}

urn:lsid:zoobank.org:act:5CDAC4E0-568D-4F4A-A72B-63AA5A4051C2

http://species-id.net/wiki/Ettchellsia_ignita

Figs $1-3,10,12$

Type series. Holotype ${ }_{+}:$"MALAYSIA: Negeri Selangor, Ulu Gombak (Univ. Malaya Field Studies Centre, 220m alt) Malaise trap, 7-11.iv.2007 T. Tsuru \& M. Maruyama leg.", "HOLOTYPE: Ettchellsia ignita Mita \& Shaw, 2012, sp. n.". Paratype: $1{ }^{\top}$, Sandan, Borneo, Baker leg. The holotype is deposited in the Systematic Entomology, Hokkaido University Museum, Sapporo, Japan (SEHU).The paratype is deposited in the U.S. National Museum of Natural History, Washington D.C, USA (NMNH).

Description. (Female) Head (Figs 1-2) $1.48 \times$ wider than long, covered with long erect setae and short decumbent setae; frons reticulate; surface around ocellar triangle smooth with rows of punctures behind anterior ocellus and outside of posterior ocelli; vertex reticulate-rugose; eye margined posteriorly by foveate groove and single postocular orbital carina; gena sparsely punctate with irregular carinae under orbital carina; occipital carina forming a small depression; clypeus transversely rugose.

Mesosoma (Fig. 3) entirely covered with short decumbent white setae, but long erect setae also present on dorsal surface; pronotum dorsally forming acute corner; mesoscutum humped, sparsely scattered with small punctures; lateral carina present on anterior mesoscutal surface; axilla and scutellum sparsely scattered with small punctures; metanotum setose; propodeum (Fig. 12) with pair of median, submedian and lateral carinae; median carina wider than other carinae, dorsal surface flattened (Fig. 12); median propodeal region narrower anteriorly, with several transverse carinae, posterior margin dorsally produced; submedian region with three transverse carinae, medially narrowed; lateral region with four transverse carinae.

Fore wing (Fig. 1) bearing four transverse dark bands; vein M $1.9 \times$ basal part of RS; erect setae on C $0.2 \times$ those on $\mathrm{Sc}+\mathrm{R}$ and A. 


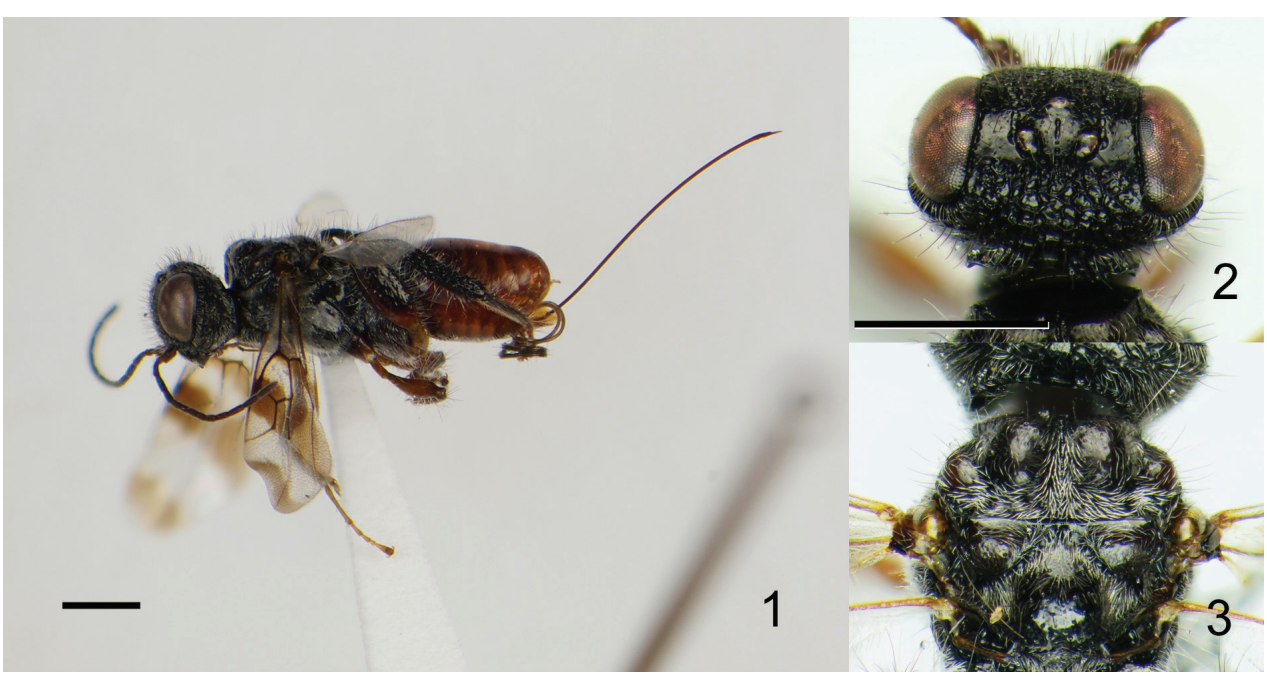

Figures I-3. Ettchellsia ignita, sp. n. (holotype). I General habitus 2 Head in dorsal view 3 Mesosoma in dorsal view. Scale $=1.0 \mathrm{~mm}$.

Metasoma smooth but anterior surface of 6th metasomal tergite and 7-8th tergites entirely strongly shagreened; ovipositor $2.00 \times$ mesosoma length, apex with small teeth and single knob.

Color. Head black; mandible black; antenna brown-black except scape and pedicel brownish; long setae on vertex and gena black, other setae white. Mesosoma black except brown tegula; long erect setae black; fore- and middle legs brown; hind leg with coxa, distal half of femur and distal four tarsomeres brown-black, trochanter and basal part of femur brown, tibia and basitarsus black; long setae on dorsal surface of hind tibia and basitarsus black. Metasoma and ovipositor reddish brown; ovipositor sheath pale brown.

Measurements. Head $1.05 \mathrm{~mm}$ long, $1.55 \mathrm{~mm}$ wide; mesosoma $2.10 \mathrm{~mm}$ long; scutum $1.35 \mathrm{~mm}$ wide; propodeal disc $0.70 \mathrm{~mm}$ long, $1.10 \mathrm{~mm}$ wide; fore wing 4.75 $\mathrm{mm}$ long; metasoma $2.30 \mathrm{~mm}$ long, $1.25 \mathrm{~mm}$ wide; ovipositor $4.20 \mathrm{~mm}$ long; total body length excluding ovipositor $5.45 \mathrm{~mm}$.

(Male) Different from female as follows: Body brownish, head dark brown with brown antenna, mesosoma brown, legs testaceous excluding brown hind tarsus, metasoma testaceous; median carinae on propodeum narrower, dorsal surface rounded (as in Fig. 13).

Measurements. Head $0.76 \mathrm{~mm}$ long, $1.24 \mathrm{~mm}$ wide; mesosoma $1.75 \mathrm{~mm}$ long; scutum $1.10 \mathrm{~mm}$ wide; propodeal disc $0.55 \mathrm{~mm}$ long, $0.90 \mathrm{~mm}$ wide; fore wing 3.55 $\mathrm{mm}$ long; metasoma $1.40 \mathrm{~mm}$ long, $0.95 \mathrm{~mm}$ wide; total body length $3.77 \mathrm{~mm}$.

Distribution. Peninsular Malaysia; Borneo.

Etymology. This species is named for the reddish coloration of the metasoma. 
Remarks. This species is similar to E. sinica with both having a strongly humped mesoscutum, however, it is distinguished from the latter by the strongly narrowed median region of propodeum (Fig. 12); long erect black setae on hind tibia and basitarsus, many of which are longer than the width of the hind tibia (setae are whitish and shorter in E. sinica); reddish brown metasoma (Fig. 1) (metasoma is black in E. sinica).

\section{Ettchellsia nigripes Mita \& Shaw, sp. $\mathrm{n}$.}

urn:lsid:zoobank.org:act:1B9CC5DE-5AAC-483A-A952-B36A84C5DDFE। http://species-id.net/wiki/Ettchellsia_nigripes

Figs 4-6, 13

Holotype $\bigcirc$ : "N. Sulawesi: Prov. Gorontalo, Pegunungan Tilongkabila, Bogani Nani Warta Bone N.P., 31. Jan-16 Feb 2010 (alt. 1200m), K. Takasuka leg. (Malaise trap)”, "HOLOTYPE: Ettchellsia nigripes Mita \& Shaw, 2012, sp. n.”. The holotype is deposited in the Laboratory of Entomology, Faculty of Agriculture, Tokyo University of Agriculture, Atsugi, Japan.

Description. (Female) Head (Figs 4-5) $1.60 \times$ wider than long, covered with long erect setae and short decumbent white setae; frons transversely rugose; surface around ocellar triangle smooth with rows of punctures behind anterior ocellus and outside of posterior ocelli; vertex reticulate-rugose; eye margined posteriorly by foveate groove and single post-ocular orbital carina; gena smooth with irregular carina under orbital carina; occipital carina not forming a depression; clypeus punctuate with small punctures.

Mesosoma (Fig. 6) entirely covered with short decumbent setae, but long erect setae also present on mesonotum; mesoscutum smooth except lateral carina present on anterior surface; dorsal mesoscutal surface not humped; axilla and scutellum smooth; metanotum setose; propodeum (Figs. 13) with pair of median, submedian and lateral carinae; median region narrowed anteriorly, with eight indistinct transverse carinae, posterior margin posteriorly produced; submedian propodeal region converging posteriorly, with two transverse carinae excluding posterior areola; lateral region with two (right side) or four (left side) transverse carinae.

Fore wing (Fig. 4) bearing four transverse dark bands but separation between second and third bands indistinct; vein M $2.2 \times$ basal part of RS; erect setae on C $1.2 \times$ longer than those on $\mathrm{Sc}+\mathrm{R}$, equal to those on vein $\mathrm{A}$.

Metasoma smooth except anterior surface of 6-8th metasomal tergites shagreened; ovipositor $1.29 \times$ mesosoma length, apex with small teeth and single knob.

Color. Body entirely black except tarsi and ovipositor sheath dark brown, ovipositor reddish brown; long setae on vertex, gena, mesonotum and dorsal surface of hind tibia and basitarsus black, other setae white.

Measurements. Head $0.80 \mathrm{~mm}$ long, $1.55 \mathrm{~mm}$ wide; mesosoma $2.15 \mathrm{~mm}$ long; scutum $1.35 \mathrm{~mm}$ wide; propodeal disc $0.75 \mathrm{~mm}$ long, $1.10 \mathrm{~mm}$ wide; fore wing 4.85 mm long; metasoma $1.90 \mathrm{~mm}$ long, $1.20 \mathrm{~mm}$ wide; ovipositor $2.75 \mathrm{~mm}$ long; total body length excluding ovipositor $4.85 \mathrm{~mm}$. 


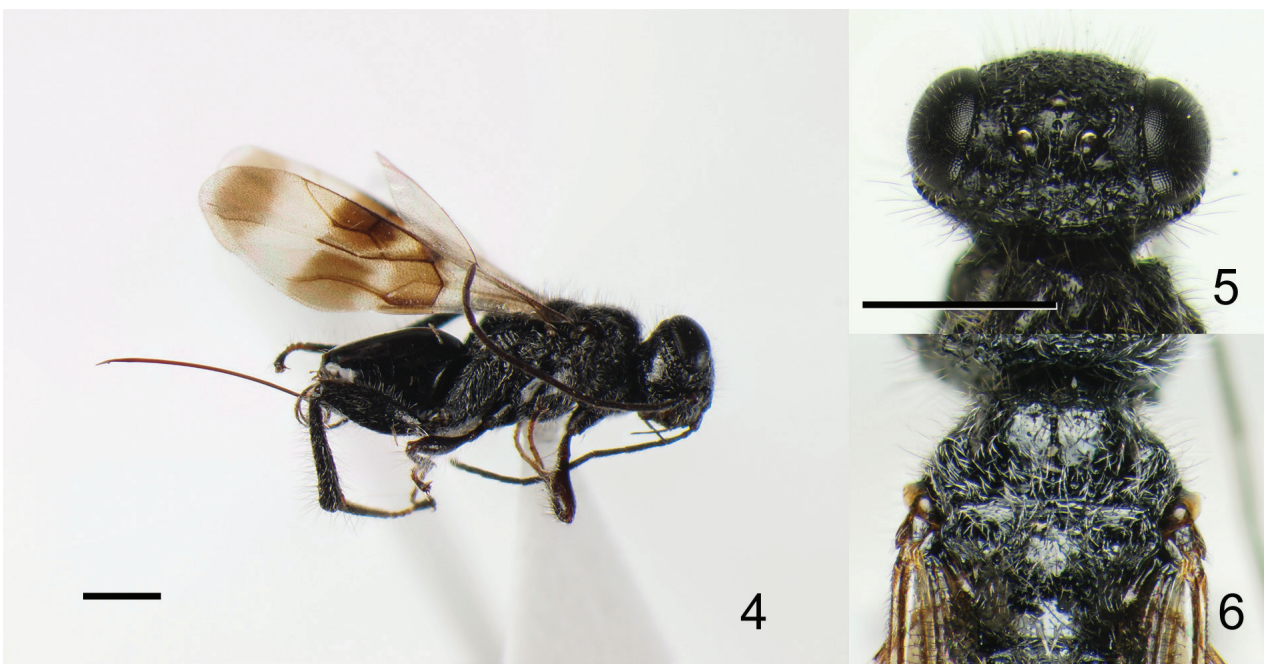

Figures 4-6. Ettchellsia nigripes, sp. n. (holotype). 4 General habitus 5 Head in dorsal view 6 Mesosoma in dorsal view. Scale $=1.0 \mathrm{~mm}$.

(Male) Unknown.

Distribution. Sulawesi Island.

Etymology. This species is named for its black legs.

Remarks. This species is similar to E. reidi Mita \& Shaw, sp. n. by the almost flat mesoscutum, however, it is distinguished from the latter by the anteriorly weakly narrowed median region of propodeum (Fig. 13) and the mostly smooth surface between posterior ocellus and eye (Fig. 5).

\section{Ettchellsia reidi Mita \& Shaw, sp. n.} urn:Isid:zoobank.org:act:403B45A6-E102-41E7-8A52-EBDC05C98BED http://species-id.net/wiki/Ettchellsia_reidi

Figs 7-9, 14

Type series. Holotype $q$ : "Sungai Sibau, nr entrance to Betung, Kerihun NP. ca. 4 km upstream, Kampung Putan. 21-27 Jun 1996, 70-90m. Chris Reid. IIS 967005”, “20 yr. old lading, closed forest, Pan traps (yellow), 1 03'13"N, 11300'56"E”, "SEM", "HOLOTYPE: Ettchellsia reidi Mita \& Shaw, 2012". Paratype: 1 , same data as above, but Pan traps (yellow) 2:9, without label of "SEM". The holotype is deposited in the Museum Zoologi Bogor (MZB), the national museum of Indonesia. The paratype is deposited in the Royal Ontario Museum (ROM) Toronto, Canada.

Description. (Female) Head (Figs 7-8) 1.53-1.56 × wider than long, covered with long erect setae and short decumbent white setae; frons transversely rugose; surface around ocellar triangle rugose with rows of punctures behind anterior ocellus and outside of posterior ocelli, but lateral row indistinct among other sculptures; vertex 


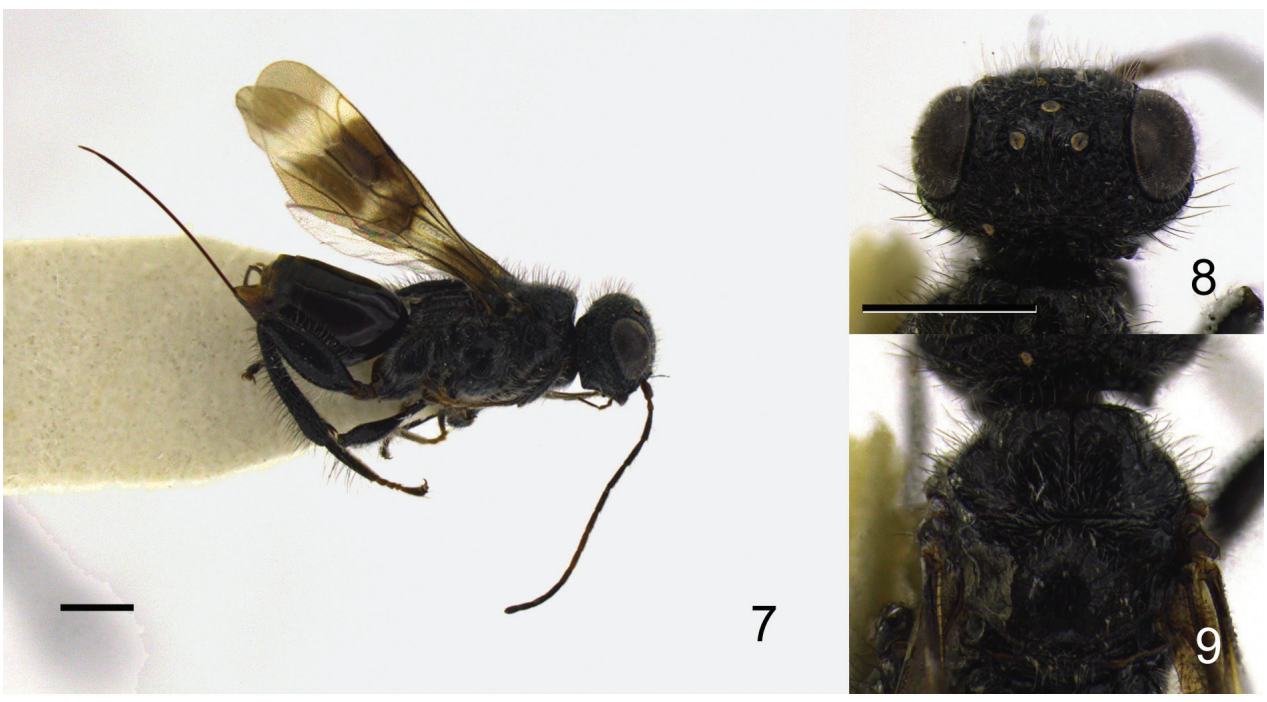

Figures 7-9. Ettchellsia reidi, sp. n. (holotype). 7 General habitus 8 Head in dorsal view 9 Mesosoma in dorsal view. Scale $=1.0 \mathrm{~mm}$.
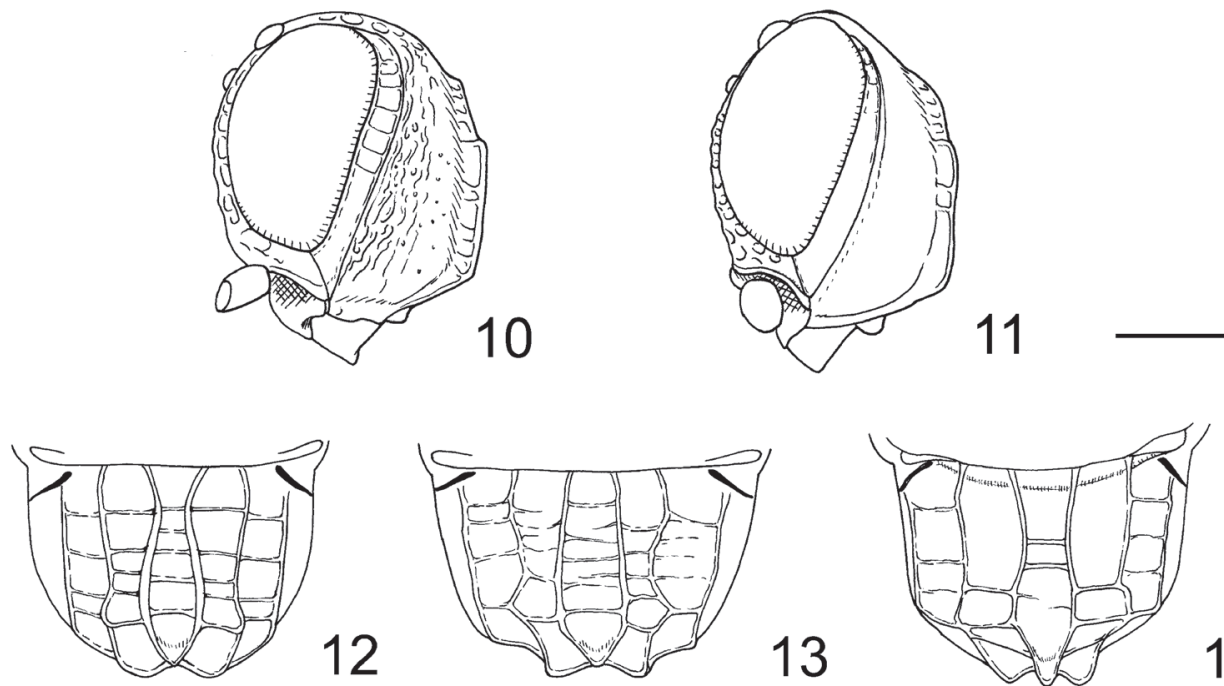

Figures I0-I4. Heads and propodea of Ettchellsia spp. I0, I2 E. ignita, sp. n. I I E. piliceps from Borneo $\mathbf{I} 3$ E. nigripes, sp. n. $\mathbf{1 4}$ E. reidi, sp. n. Scale $=0.5 \mathrm{~mm}$.

reticulate rugose; eye margined posteriorly by foveate groove and single post-ocular orbital carina; gena smooth with irregular carinae under orbital carina; occipital carina dorso-laterally with a small depression; clypeus smooth.

Mesosoma (Fig. 9) entirely covered with short decumbent setae, but long erect setae also present on mesonotum; mesoscutum smooth; lateral carina present on anterior 
surface; dorsal surface not swollen; axilla and scutellum smooth; metanotum setose; propodeum (Fig. 14) with pair of median, submedian and lateral carinae; median propodeal region narrower centrally, with a few transverse carinae in holotype, carinae indistinct in a paratype; posterior margin dorsally producing; submedian region smooth excluding posterior areola, parallel-sided; lateral region with at most four transverse carinae but keels sometimes indistinct.

Fore wing (Fig. 7) bearing three transverse dark bands with clear spot around Rs+M; vein M 1.6-1.7 × basal part of RS; erect setae on C $2.0 \times$ those on $\mathrm{Sc}+\mathrm{R}, 1.2 \times$ those on A.

Metasoma smooth except anterior surface of $6-8$ th metasomal tergites shagreened; ovipositor 1.34-1.45 $\times$ mesosoma length, apex with small teeth and single knob.

Color. Head black; mandible dark brown; antenna dark brown except scape, pedicel and 5-8th flagellomeres brown; long setae on vertex, gena black. Mesosoma black; long setae on mesonotum black; legs black except tarsi brownish; long setae on dorsal surface of hind tibia and basitarsus black. Metasoma brownish black; ovipositor and ovipositor sheath dark brown.

Measurements. Head 0.80-1.10 mm long, 1.25-1.65 mm wide; mesosoma 1.55$2.20 \mathrm{~mm}$ long; scutum $0.95-1.30 \mathrm{~mm}$ wide; propodeal disc $0.65-0.85 \mathrm{~mm}$ long, 0.85-1.10 mm wide; fore wing 3.50-4.90 mm long; metasoma $1.55-2.35 \mathrm{~mm}$ long, $0.85-1.25 \mathrm{~mm}$ wide; ovipositor $2.25-2.95 \mathrm{~mm}$ long; total body length excluding ovipositor $3.75-5.45 \mathrm{~mm}$.

(Male) Unknown.

Distribution. Kalimantan Barat, Borneo.

Etymology. The species name is dedicated to the collector of the types, Chris Reid, a coleopterist working at the Australian Museum, Sydney.

Remarks. The specimens were collected as part of the Insects of Indonesia Project, a collaboration of the ROM with the MZB. This species is similar to E. nigripes Mita \& Shaw, sp. n. About identification of the two species, see the remarks of E. nigripes.

\section{Acknowledgments}

The authors express cordial thanks to Dr Keizo Takasuka and Mr Kyohei Watanabe from Kobe University, Kobe, Japan for providing the holotype of Ettchellsia nigripes sp. n. We are also grateful to Dr Christopher Darling (ROM), Dr David Smith (NMNH) and Mr. Takuma Yoshida (SEHU) for loaning the type and non-type material. Ms Natalie Dale-Skey Papilloud, Natural History Museum, London, UK loaned an identified female specimen of E. piliceps for comparison.

\section{References}

Baltazar CR (1961) Ettchellsia philippinensis sp. nov. (Dinapsinae, Megalyridae, Hymenoptera). The Philippine Journal of Science 90: 219-220. 
Cameron P (1909) Description of a new genus and species of parasitic Hymenoptera, representing a new tribe, from Kuching, Borneo. Deutsche entomologische Zeitschrift 1909: 208-209.

He J-H (1991) A new species of the genus Ettchellsia Cameron (Hymenoptera: Ichneumonoidea, Megalyridae). Acta Entomologica Sinica, 34(4), 475-477.

Mita T, Konishi K, Terayama M, Yamane S (2007) Two new species of the genus Carminator Shaw from Japan, the northernmost record of extant Megalyridae (Hymenoptera). Entomological Science 10: 201-208. doi: 10.1111/j.1479-8298.2007.00213.x

Mita T, Konishi K (2011) Biogeography and phylogeny of Carminator (Hymenoptera: Megalyridae). Systematic Entomology 36: 569-581. doi: 10.1111/j.1365-3113.2010.00548.x

Shaw SR (1988) Carminator, a new genus of Megalyridae (Hymenoptera) from the Oriental and Australian regions, with a commentary of the definition of the family. Systematic Entomology 13: 101-113. doi: 10.1111/j.1365-3113.1988.tb00233.x

Shaw SR (1990) Phylogeny and biogeography of the parasitoid wasp family Megalyridae (Hymenoptera). Journal of Biogeography 17: 569-581. doi: 10.2307/2845141

Shaw SR, van Noort S (2009) A new Dinapsis from the Central African Republic (Hymenoptera, Megalyridae, Dinapsini). Zootaza 2118: 30-36.

Vilhelmsen L, Perrichot V, Shaw SR (2010) Past and present diversity and distribution in the parasitic wasp family Megalyridae (Hymenoptera). Systematic Entomology 35: 658-677. doi: $10.1111 / \mathrm{j} .1365-3113.2010 .00537 . x$

Waterson J (1922) A new family of Hymenoptera from South Africa. Annals and Magazine of Natural History 9(10): 418-420. doi: 10.1080/00222932208632792 